\title{
Neonatal heart transplantation
}

\author{
Mohan John, Leonard L. Bailey \\ Department of Cardiovascular and Thoracic Surgery, Loma Linda University Children's Hospital, Loma Linda, California, USA \\ Correspondence to: Leonard L. Bailey, MD. Department of Cardiovascular and Thoracic Surgery, Loma Linda University Children's Hospital, 11175 \\ Campus St, Suite 21120, Loma Linda, CA 92354, USA. Email: 1lbailey@1lu.edu.
}

\begin{abstract}
Neonatal heart transplantation was developed and established in the 1980's as a durable modality of therapy for complex-uncorrectable heart disease. Patients transplanted in the neonatal period have experienced unparalleled long-term survival, better than for any other form of solid-organ transplantation. However, the limited availability of neonatal and young infant donors has restricted the indications and applicability of heart transplantation among newborns in the current era. Indications for heart transplantation include congenital heart disease not amenable to other forms of surgical palliation, and cardiomyopathy, including some primary tumors. Use of ABO-incompatible transplants, and organs with prolonged cold ischemic time or marginal function have all been associated with good outcomes in infants. These extended strategies to increase the donor pool may also someday include donation after determination of circulatory death and the use of anencephalic donors. The operative techniques for donors and recipients of neonatal heart transplantation are unique and have been well-described. Immunosuppression protocols for neonates need not include induction and are largely steroid-free. Newborn and young infant transplant recipients have fewer episodes of rejection, less coronary allograft vasculopathy, less post-transplant lymphoproliferative disease and less renal dysfunction than their older counterparts. Long-term outcomes have been very encouraging in terms of graft survival, patient survival, and quality of life. Our review highlights the history, current indications, techniques and outcomes of heart transplantation in this immunologically-privileged subset of patients.
\end{abstract}

Keywords: Heart transplantation; neonatal immunity; heart failure; congenital heart defect

Submitted Aug 23, 2017. Accepted for publication Dec 11, 2017.

doi: 10.21037/acs.2018.01.05

View this article at: http://dx.doi.org/10.21037/acs.2018.01.05

\section{Introduction}

Neonatal heart transplantation was developed and established in the 1980's as a durable modality of therapy for complex, uncorrectable congenital heart disease. Patients transplanted in the neonatal period have experienced unparalleled long-term survival $(1,2)$. In the modern era, however, the restrictive donor pool, along with improvement in early and intermediate outcomes of palliative reconstructive procedures, have markedly reduced the number of neonates undergoing heart transplantation worldwide. We sought to review the history and development of heart transplantation for neonates, current indications and technical challenges, long-term outcomes and complications, and finally potential strategies to increase the limited donor pool.

\section{Neonatal immune tolerance}

The immune system of the newborn is immunologically naive and presents a unique 'window of opportunity' for successful allotransplantation. Billingham et al. first demonstrated that the administration of hematopoietic cells to mice in early life allowed subsequent allogeneic grafts to be retained (3). The mechanism of neonatal tolerance cannot be entirely attributed to deletion of alloreactive T-cells, but is probably multifactorial and a comprehensive understanding remains elusive. Other potential mechanisms include the interaction between donor and host cells to regulate the immune response, an immature antigenpresenting system, and low level cellular chimerism (4).

It is possible that careful manipulation of one or more of these potential mechanisms of tolerance could lead to 
the holy grail of transplantation-long-term graft survival without immunosuppression. As it is, human fetuses start exhibiting self-recognition in cell-mediated cytotoxicity assays after 18 weeks of gestation. Their immune response, while somewhat more tolerant in nature, is well-established by the time of birth (5). This would suggest that humanterm neonates are beyond the stage of true graft-specific tolerance induction. Fetal cardiac anomalies requiring transplantation can be diagnosed on ultrasonography by mid-gestation, but obtaining and infusing allograft donor-specific tolerizing cells into the fetus at this stage is not feasible. Recent reports have highlighted the role of impaired B-cell immunity to carbohydrate antigens, discarded infant thymus as a source of regulatory T-cells, and suppressive erythroid precursors as potential targets for tolerance induction beyond early infancy (4).

In clinical practice, neonatal recipients of heart transplantation have demonstrated better survival, a lower immunosuppressive burden, and less chronic rejection than their older counterparts (6). In 2001, West et al. showed that $\mathrm{ABO}$-incompatible heart transplants could be safely performed in infants due to the inability of the immature immune system to mount an effective response to carbohydrate antigens (7). ABO-incompatible heart transplantation has since increased access to heart transplantation in the first two years of life with equivalent long-term outcomes as seen in ABO-compatible transplants.

\section{History and current status}

In New York in 1967, Adrian Kantrowitz, after considerable experimental work with puppies, first attempted clinical neonatal heart transplantation. The recipient was a 19-day old baby with severely symptomatic Ebstein's anomaly (8). The donor was anencephalic. Sadly, that newborn recipient suffered cardiac arrest and died 6.5 hours after the procedure. The cause of cardiac arrest was never clearly identified, and Kantrowitz did not pursue heart transplantation.

Hypoplastic left heart syndrome (HLHS), a much more common and uniformly fatal congenital heart anomaly, had not yet been addressed surgically. The naive immune system of a neonate presented a unique opportunity, however, to reconsider heart transplantation in this group of patients. In 1978, we began studying animal (goat) models of neonatal heart allo- and xeno-transplantation. With the introduction of the immunoregulatory agent cyclosporine-A in 1981, neonatal allograft recipients experienced normal maturation and reproductive capability and survival into late adulthood. Recipients of xenografts also had unprecedented survival (9). In addition, we investigated the use of immature baboons as potential clinical donors. We tested their immunologic compatibility with human neonates using human leukocyte antigen (HLA) typing, two-way mixed lymphocyte cultures and $e x$-vivo perfusion studies. Encouraged by our results, we sought and eventually obtained institutional approval for clinical neonatal xenotransplantation. In July of 1984, Sir Magdi Yacoub and his team in London performed cardiac allotransplantation in an 11-day old baby with HLHS. She developed multiple postoperative complications from which she died after 18 days. In October 1984, we performed orthotopic cardiac xenotransplantation on 'Baby Fae', a 12-day old neonate with HLHS. The donor was an immunologically-selected infant female baboon. The recipient baby recovered well initially, but died 20 days later. Her causes of death were not well understood, but did include widespread systemic vascular hemagglutination and multi-organ micro-infarction. This devastating outcome likely related to a bedside error in medication delivery (10). Humoral and cellular rejection of the xenograft were not apparent. Neonatal xenotransplantation would not be attempted again, but our experience with Baby Fae led to the first successful neonatal heart transplant just over a year later on November 20, 1985. That recipient remains alive and well with the same donor heart to this day. He is gainfully employed.

Heart transplantation in neonates and infants became well-established in the subsequent decade. Still, this new therapy was limited by the donor pool, which seldom exceeded 70-100 hearts per year in North America. The development of primary transplantation for treatment of HLHS occurred in parallel with staged, palliative reconstruction being pioneered by William Norwood in Boston and, subsequently, in Philadelphia. As more surgical teams gained experience with Norwood's strategy, refinements in operative technique and perioperative management resulted in competitive early outcomes for patients with single ventricle anomalies. Primary heart transplantation for HLHS and equivalent malformations was then held in reserve for those infants deemed unsuitable for the Norwood-Fontan pathway.

Neonatal heart transplants accounted for $20 \%$ of all pediatric transplants from 1993 to 2009. Today, there are seldom more than three or four newborns on the waiting list at any given time in the Unites States. The average wait-time is 110 days. Wait-list mortality for newborns 


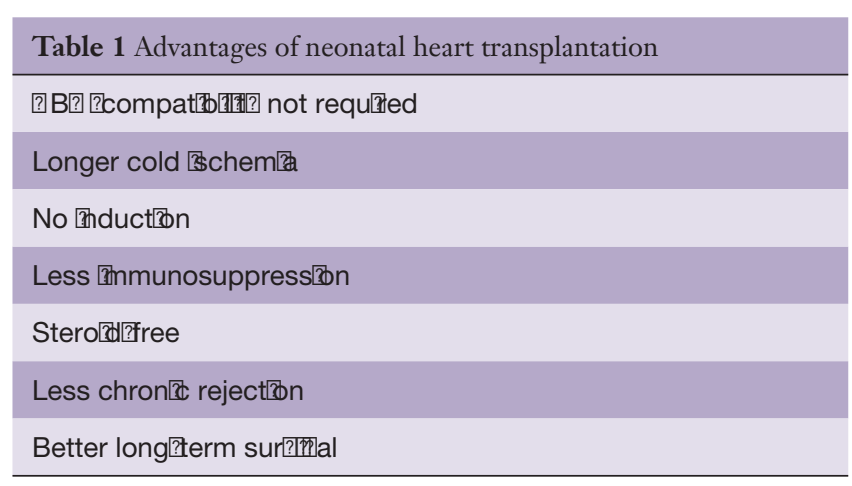

is $34 \%$. Currently, only about 8-12 newborn transplants are performed in the United States each year (11). The unique characteristics of newborn heart transplantation are summarized in Table 1.

\section{Indications for newborn heart transplantation}

Current indications for primary transplantation for endstage heart disease in very early life are as follows (12):

(I) Severe cardiomyopathy, including some primary cardiac tumors;

(II) Single ventricle physiology (e.g., HLHS) with reduced ventricular function and severe atrioventricular valve insufficiency;

(III) Pulmonary atresia-intact ventricular septum with right ventricle dependent coronary circulation;

(IV) Severe Ebstein's anomaly not amenable to surgical repair or palliation.

\section{Contraindications to heart transplantation in very early infancy}

(I) Any non-cardiac condition (genetic. somatic) that significantly shortens life expectancy;

(II) Active systemic infection;

(III) All forms of cancer;

(IV) Renal, hepatic, or other gastrointestinal dysfunction not responsive to medical therapy;

(V) Major neurological compromise.

\section{Donor evaluation}

Guidelines for determination of brain death in young infants and term newborns were clarified by the American Academy of Pediatrics in 2011 (13). The diagnosis is essentially clinical. It requires a neurological examination, apnea testing and a period of clinical observation. Ancillary tests (electroencephalogram and radionuclide cerebral blood flow) may be used based on the physician's discretion in equivocal cases. The size and function of the donor organ, the projected cold ischemic time, and screening for donor infections, are each important consideration in heart transplantation at any age. Weight is used to identify and select potential recipients for a given donor. Donor weight generally reflects the heart size in the pediatric population. Among adults, the recommendation is for the donor weight to be within $30 \%$ of recipient weight in order to ensure appropriate size-matching (14). The use of over or undersized grafts is not recommended in the adult population. In children, however, the use of oversized grafts (donor-recipient weight ratio $>2.5$ ) has produced comparable outcomes to well matched grafts (15). The geographic distance of the donor from the transplantation center rarely limits the availability of donors for infant recipients. Cold ischemic times should always be minimized, but durations up to 8 and 10 hours have been reported with success (16). The duration of cold ischemic time does have an effect on chronic graft survival, but this effect is less apparent among pediatric recipients (17).

Donors are screened for human immunodeficiency virus, hepatitis B, hepatitis C, Epstein-Barr virus and cytomegalovirus. Active infection with the first three of these viruses is a contraindication to use of the donor organ. The ideal donor heart will have normal ventricular function and no structural abnormality. However, the utilization of hearts with diminished function in the donor has been shown to be well-tolerated in the pediatric population. Correctable heart defects like atrial septal defect and ventricular septal defect are not contraindications to donor graft utilization. As discussed earlier, studies indicate good outcomes with ABO-incompatible heart transplants in infants (7). This strategy has increased donor utilization, but has not resulted in a decrease in waiting-list mortality in the United States.

Donor heart ejection fraction persistently below 25\%, severe congenital heart disease, prolonged cardiac arrest (>45-60 minutes), and severe systemic illness or active infection are all contraindications to infant heart donation.

\section{Donor organ availability}

Infants requiring heart transplantation have the highest waiting-list mortality of any age group (18). Approximately 
Table 2 Potential strategies to increase the availability of donors for neonatal transplantation

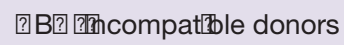

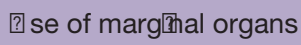

[? onat?n

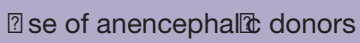

?enotransplantat?on

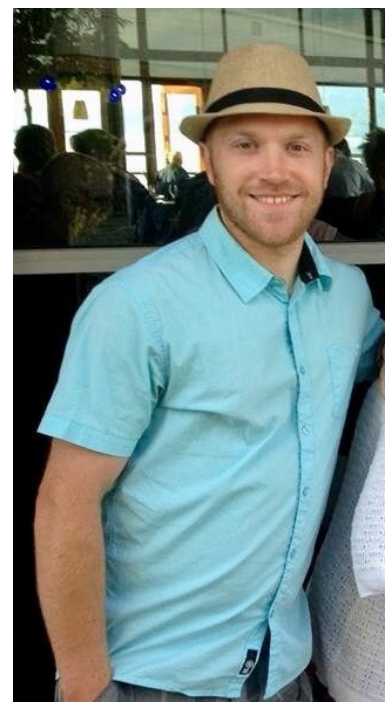

Figure 1 Paul Holc-youngest surviving recipient at 3 hours of age. Now 28 years old and the father of a healthy son (Photo reproduced by permission).

one-quarter of infants will die before they receive a transplant (19). The number of available donors has been relatively static, while the number on the waiting list continues to gradually increase every year. Two public health measures have reduced the number of available donors: the recognition of the importance of sleep positioning to prevent sudden infant death syndrome, and mandatory infant car seats to prevent injury (6). Recent studies have examined methods to expand the donor pool and minimize the non-utilization of functional organs (20). Donation after determination of circulatory death was shown to be feasible in infants, but has not yet been widely adopted due to ethical and legal constraints. The use of organs from neonates with anencephaly has been explored. However, uniform criteria must be established in order to ethically justify the use of this subgroup of patients as organ donors (Table 2).

\section{Fetal listing}

Fetal listing of patients with HLHS and its variants for heart transplantation can potentially decrease time on the waiting list. United Network for Organ Sharing (UNOS) allowed for fetal listing beyond 32 weeks gestation, but has since changed their policy and eliminated this option in 2016. Between 1993 and 2009, fetuses comprised one percent of children listed for transplantation. A higher proportion of fetal-listed patients had undergone transplant with a lower wait-list mortality as compared to neonates (21). Figure 1 is of a recipient who was listed as a fetus.

\section{Evaluation of neonatal recipient}

The pre-transplant workup of neonates and infants with congenital heart disease is very similar to that for adults, with a few caveats. Potential recipients must be carefully screened for additional life-limiting congenital malformations. Neurological anomalies and injuries may be difficult to detect clinically in the newborn. If suspected, head ultrasound and electroencephalography can be performed before proceeding to computed tomography and magnetic resonance imaging. Hepatic and renal function tests form part of the pre-transplant workup. The development of elevated and fixed pulmonary vascular resistance requires time; hence, pulmonary vascular disease is rarely, if ever, an issue in very early life. Panel-reactive antibody testing is performed to assess for the presence of antibodies against HLAs. An assessment of the family support system should be made by a social worker prior to consideration for transplant.

\section{Operative techniques}

Donor procedure: the donor operation for neonatal transplantation has been standardized (22). Preliminary dissection is achieved prior to heparinization. The entire aortic arch is included for recipients with hypoplastic arch. En-bloc removal of additional systemic vein or pulmonary artery may be utilized for reconstruction in recipients with situs inversus. Graft preservation is accomplished using gravity-fed cold crystalloid cardioplegia solution, and transport for storage is accomplished in iced 5\% dextrose in normal saline. 
Recipient procedure: the now standard bicaval-left atrial technique is used for implantation. Deep hypothermia with low-flow perfusion facilitates the procedure. Arch reconstruction is accomplished using donor aorta and a brief period of hypothermic circulatory arrest or selective cerebral perfusion (23). A left-sided superior vena cava is anastomosed to donor innominate vein. Pulmonary artery reconstruction, repair of anomalous pulmonary venous connection, and adaptation of the new heart to positional abnormalities of the native heart are additional technical challenges in transplantation for congenital heart disease (12). Some centers perform arch and pulmonary artery reconstruction with commercial materials prior to implantation to reduce the warm ischemia time (24). This is unnecessary for primary transplant reconstruction in early life.

\section{Immunosuppression}

Long-term immunosuppression following heart transplantation in children is achieved with a calcineurin inhibitor, an anti-proliferative agent and frequently, a corticosteroid. Most pediatric centers advocate induction therapy in the perioperative period with antithymocyte globulin or an IL-2 receptor antagonist. Our immunosuppression protocol has been described elsewhere (6). We do not use induction therapy for neonatal recipients. Our goal is the avoidance of long-term steroids. Less than five percent of our infant transplants required maintenance steroid therapy. Diagnosed graft rejection is reversed with intravenous corticosteroid and an increase in maintenance immunoregulation. Anti-thymocyte globulin (five doses) may be added for recalcitrant or recurrent rejection.

\section{Graft surveillance}

The gold standard for graft surveillance is endomyocardial biopsy. Non-invasive methods of surveillance (primarily echocardiography) are widely used, but are associated with limited sensitivity. There is significant variation among transplant centers with respect to surveillance protocols in children (25). Due to the lower incidence of rejection in the current era, the need for frequent endomyocardial biopsy has been questioned. Endomyocardial biopsy is largely unnecessary among recipients in very early life. Annual coronary angiography and, later in life, intravascular ultrasound are utilized to help monitor the development and progression of cardiac allograft vasculopathy.

\section{Complications}

Rejection is one of the leading causes of mortality following pediatric heart transplantation. Sixty-five percent of patients will experience rejection at least once during their lifetime, 22 percent within the first year following transplant (2). Early rejection episodes and significant late rejection (>1 year) have both been shown to correlate with decreased long-term survival. The use of induction therapy has not clearly reduced episodes of rejection (2). An episode of moderate rejection is treated with an increase in the dosage of immunosuppression. Episodes of rejection producing graft dysfunction require intravenous corticosteroid therapy and/or anti T-cell antibodies. Neonatal recipients usually exhibit a less intense immune response to their graft.

Cardiac allograft vasculopathy (CAV) is the leading cause of late graft loss in long-term survivors following heart transplantation. The incidence of CAV was found to be $25 \%$ at 10 years and $54 \%$ at 15 years following pediatric transplantation. With regard to neonatal recipient outcomes, the incidence of CAV was lowest in the infant age group and higher with increasing age (26). The use of mechanistic target of rapamycin (m-TOR) inhibitors has shown promise in the prevention and treatment of CAV. Treatment for progressive disease is re-transplantation. Much less commonly encountered focal proximal stenoses are treated with coronary artery stenting.

Post-transplant lymphoproliferative disease (PTLD) is the most common malignancy that develops after heart transplantation and is not uncommon in children. Most cases are related to EBV infection. The incidence of PTLD is about $10 \%$ at 10 years in pediatric recipients (27). In our series of infant (0-12 months) heart transplants, a 22 percent actuarial risk of PTLD was observed at 25 years (6). There is a strong effect of age on development of PTLD. Young infants and older children ( $>10$ years) have a lower incidence of PTLD compared to young children (1-10 years) (27). The protective influence of maternal antibodies in infants may help explain this finding. Despite treatment with chemotherapy and/or reduction of immunosuppression, the prognosis for PTLD remains poor.

Renal dysfunction post-transplant is primarily due to use of calcineurin inhibitors. Patients who received transplants as infants had a lower incidence of severe renal dysfunction as compared to older children, four percent in 10 years (28). Thirteen percent of long-term survivors following pediatric transplant ultimately required chronic 


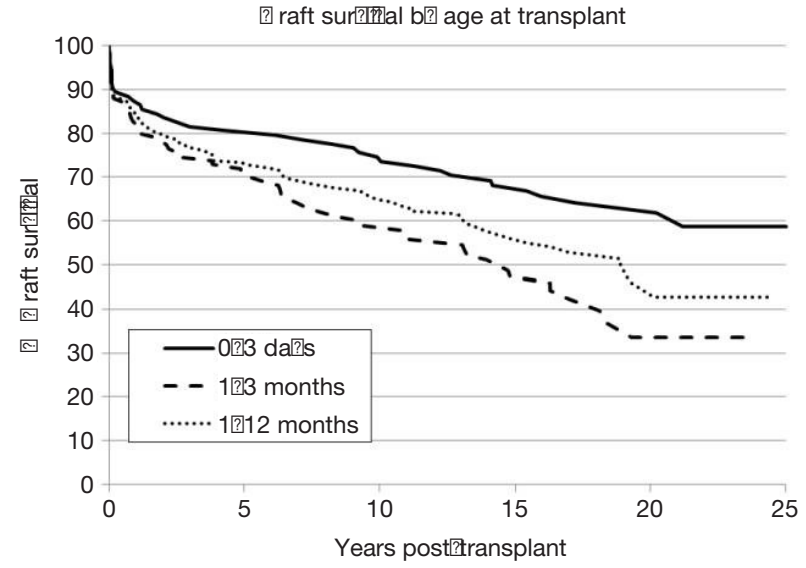

Figure 2 Actuarial graft survival for 0-30 days old heart transplant recipients versus 1-3 months old recipients (1985 to 2010, Loma Linda data) (4).

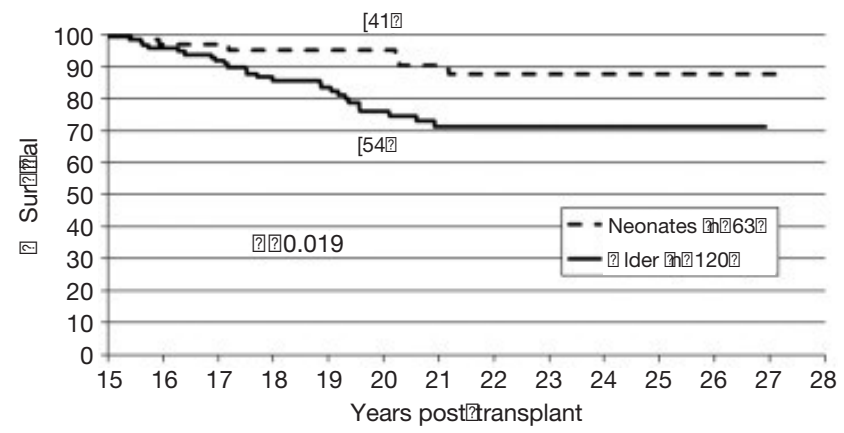

Figure 3 Long-term survival after pediatric heart transplantation, neonates $v s$. other recipients (1985-1998, Loma Linda data) (28).

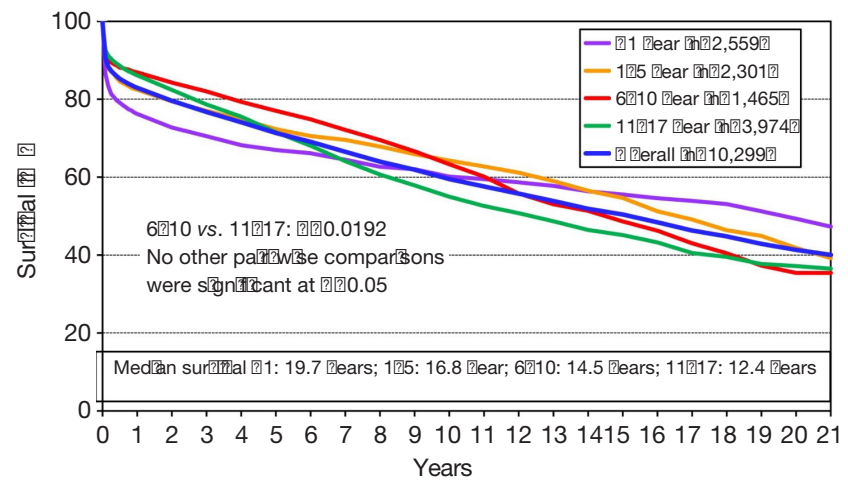

Figure 4 Kaplan-Meier survival for pediatric heart transplant recipients (1982 to 2011, ISHLT data) (15). hemodialysis (1).

\section{Outcomes}

The long-term outcomes for neonatal heart transplantation have not been well-documented in the literature, owing to the limited number of cases performed. Chinnock et al. included neonates in his report on the Loma Linda infant experience in 2011 (6). Heart transplants were accomplished in 103 neonates from 1985 to 2010. The indication for surgery in most of these patients was HLHS or its equivalent; virtually all of these recipients had congenital heart disease. Newborn recipients of heart transplants had better late survival than older infants. The actuarial graft survival was $59 \%$ at 25 years (Figure 2). When pediatric transplant recipients who survived beyond 15 years were reviewed, those who received transplants within the first 30 days of postnatal life had a clear longterm survival advantage (Figure 3) (1). These findings may be due to the perceived advantages of the immature recipient immune system, less intense immunosuppression, and avoidance of complications related to wait-list time. The International Society for Heart and Lung Transplantation also confirmed that infants had higher late survival following heart transplant as compared to other pediatric age groups (Figure 4). Median survival was 19.7 years for infants aged 12 months or less, 16.8 years for children between 1 and 5 years of age, 14.5 years for children between the ages of 6 and 10 years, and 12.4 years for adolescents (14). Reported neurodevelopmental outcomes for pediatric transplant recipients have been encouraging. A majority of long-term survivors (95\%) were enrolled in or had completed high school and $53 \%$ of them had, at minimum, started college (1).

\section{Limitations}

There are a limited number of neonatal heart transplants that are performed worldwide every year, owing to a restricted donor pool. Hence, current literature is lacking in articles focused specifically on neonatal heart transplantation. Much of the data gathered for this review was obtained from single-center retrospective studies and subset analyses of large national and international 
databases.

\section{Conclusions}

Heart transplantation remains the most definitive modality of treatment for infants and children with end-stage heart disease, whether congenital or myopathic. Longterm outcomes following heart transplantation during neonatal life are better than for any other form of solid organ transplantation. Late survivors have been productive in society and have a good quality of life. The limited number of available donors has restricted the indications and applicability of transplantation in very early infancy. Efforts to expand the donor pool to include donation after circulatory determination of death, using hearts with marginal function, and use of anencephalic donors may help reduce the shortfall in supply of these exceptional organs. Perhaps a renewed interest in xenotransplantation may be the answer to this conundrum, although clinical trials await additional research and regulatory approval.

\section{Acknowledgements}

None.

\section{Footnote}

Conflicts of Interest: The authors have no conflicts of interest to declare.

\section{References}

1. Copeland H, Razzouk A, Chinnock R, et al. Pediatric recipient survival beyond 15 post-heart transplant years: a single-center experience. Ann Thorac Surg 2014;98:214550; discussion 2150-1.

2. Dipchand AI, Kirk R, Edwards LB, et al. The Registry of the International Society for Heart and Lung Transplantation: Sixteenth Official Pediatric Heart Transplantation Report--2013; focus theme: age. J Heart Lung Transplant 2013;32:979-88.

3. Billingham RE, Brent L, Medawar PB. Actively acquired tolerance of foreign cells. Nature 1953;172:603-6.

4. West LJ. Neonatal tolerance. Curr Opin Organ Transplant 2016;21:66-73.

5. Brent L. Neonatal tolerance: the biologic and historic background of exploiting the immature immune system for transplantation. Transplantation 2014;98:1248-52.
6. Chinnock RE, Bailey LL. Heart transplantation for congenital heart disease in the first year of life. Curr Cardiol Rev 2011;7:72-84.

7. West LJ. ABO-incompatible hearts for infant transplantation. Curr Opin Organ Transplant 2011;16:548-54.

8. Kantrowitz A, Haller JD, Joos H, et al. Transplantation of the heart in an infant and an adult. Am J Cardiol 1968;22:782-90.

9. Bailey LL. The evolution of infant heart transplantation. J Heart Lung Transplant 2009;28:1241-5.

10. Bailey LL. Baboon-to-human cardiac xenotransplantation in a neonate. JAMA 1985;254:3321-9.

11. Chen J. Indications and Strategies of Neonatal Heart Transplantation. STS Annual Meeting, 2011.

12. Kirklin JK. Current challenges in pediatric heart transplantation for congenital heart disease. Curr Opin Organ Transplant 2015;20:577-83.

13. Nakagawa TA, Ashwal S, Mathur M, et al. Clinical report-Guidelines for the determination of brain death in infants and children: an update of the 1987 task force recommendations. Pediatrics 2011;128:e720-40.

14. Costanzo MR, Dipchand A, Starling R, et al. The International Society of Heart and Lung Transplantation Guidelines for the care of heart transplant recipients. J Heart Lung Transplant 2010;29:914-56.

15. Razzouk AJ, Johnston JK, Larsen RL, et al. Effect of oversizing cardiac allografts on survival in pediatric patients with congenital heart disease. J Heart Lung Transplant 2005;24:195-9.

16. Scheule AM, Zimmerman GJ, Johnston JK, et al. Duration of graft cold ischemia does not affect outcomes in pediatric heart transplant recipients. Circulation 2002;106:I163-7.

17. Russo MJ, Chen JM, Sorabella RA, et al. The effect of ischemic time on survival after heart transplantation varies by donor age: an analysis of the United Network for Organ Sharing database. J Thorac Cardiovasc Surg 2007;133:554-9.

18. Almond CSD, Thiagarajan RR, Piercey GE, et al. Waiting list mortality among children listed for heart transplantation in the United States. Circulation 2009;119:717-27.

19. Mah D, Singh TP, Thiagarajan RR, et al. Incidence and risk factors for mortality in infants awaiting heart transplantation in the USA. J Heart Lung Transplant 2009;28:1292-8.

20. Sarnaik AA. Neonatal and pediatric organ donation: ethical perspectives and implications for policy. Front Pediatr 
2015;3:100.

21. Conway J, Chrisant MRK, West LJ, et al. Outcomes of fetal listed patients awaiting heart transplantation. Pediatr Transplant 2013;17:653-60.

22. Bailey L, Concepcion W, Shattuck H, et al. Method of heart transplantation for treatment of hypoplastic left heart syndrome. J Thorac Cardiovasc Surg 1986;92:1-5.

23. Vricella LA, Razzouk AJ, del Rio M, et al. Heart transplantation for hypoplastic left heart syndrome: modified technique for reducing circulatory arrest time. J Heart Lung Transplant 1998;17:1167-71.

24. Iyengar AJ, Sharma VJ, Weintraub RG, et al. Surgical strategies to facilitate heart transplantation in children after failed univentricular palliations: the role of advanced intraoperative surgical preparation. Eur J Cardiothorac
Surg 2014;46:480-5.

25. Godown J, Harris MT, Burger J, et al. Variation in the use of surveillance endomyocardial biopsy among pediatric heart transplant centers over time. Pediatr Transplant 2015;19:612-7.

26. Kobayashi D, Du W, L'ecuyer TJ. Predictors of cardiac allograft vasculopathy in pediatric heart transplant recipients. Pediatr Transplant 2013;17:436-40.

27. Chinnock R, Webber SA, Dipchand AI, et al. A 16-year multi-institutional study of the role of age and EBV status on PTLD incidence among pediatric heart transplant recipients. Am J Transplant 2012;12:3061-8.

28. Thrush PT, Hoffman TM. Pediatric heart transplantationindications and outcomes in the current era. J Thorac Dis 2014;6:1080-96.
Cite this article as: John M, Bailey LL. Neonatal heart transplantation. Ann Cardiothorac Surg 2018;7(1):118-125. doi: 10.21037/acs.2018.01.05 